\title{
Molecular Dynamics Modellization and Simulation of Water Diffusion through Plant Cutin
}

\author{
Antonio Matas and Antonio Heredia* \\ Departamento de Biología Molecular y Bioquímica, Facultad de Ciencias, Universidad de \\ Málaga, E-29071 Málaga, Spain. Fax: +34-952-132000. E-mail: heredia@uma.es \\ * Author for correspondence and reprint requests \\ Z. Naturforsch. 54c, 896-902 (1999); received April 12/May 11, 1999 \\ Cuticle, Cutin, Water Diffusion, Molecular Modellization, Molecular Dynamics \\ A theoretical molecular modelling study has been conducted for cutin, the biopolyester \\ that forms the main structural component of the plant cuticle. Molecular dynamics (MD) \\ simulations, extended over several ten picoseconds, suggests that cutin is a moderately flexi- \\ ble netting with motional constraints mainly located at the cross-link sites of functional ester \\ groups. This study also gives structural information essentially in accordance with previously \\ reported experimental data, obtained from X-ray diffraction and nuclear magnetic resonance \\ experiments. MD calculations were also performed to simulate the diffusion of water mole- \\ cules through the cutin biopolymer. The theoretical analysis gives evidence that water perme- \\ ation proceedes by a "hopping mechanism". Coefficients for the diffusion of the water mole- \\ cules in cutin were obtained from their mean-square displacements yielding values in good \\ agreement with experimental data.
}

\section{Introduction}

Aerial parts of higher plants are covered by a continuous extra-cellular layers, the cuticle. The main function ascribed to the cuticle is to minimise water loss and, in addition, it limits the loss of substances from plant internal tissues and also protects the plant against physical, chemical and biological impacts (Holloway, 1982).

Cuticles of higher plants are chemically heterogeneous in nature, basically consisting of a wax fraction, soluble in common organic solvents, and an insoluble cuticular matrix, that forms the framework of the cuticle. This cuticular matrix is mainly formed by the biopolymer cutin, a highmolecular weight polyester composed of various inter-esterified $\mathrm{C}_{16}$ and $\mathrm{C}_{18}$ hydroxyalkanoic acids (Walton, 1990). The ester bonds in cutin can be cleaved by alkaline hydrolysis to yield the corresponding hydroxyfatty acids. Further compositional analyses have established a few classes of monomers which are present in cutin. Depolymerisation products of many plant cutins are composed almost exclusively of derivatives of the $C_{16}$ family of monomer acids, in which 10,16- and/or 9,16-dihydroxyhexadecanoic acids are the major compounds (Walton, 1990; Kolattukudy, 1996), contrasting the fact that relatively few cutins con- tain significant amounts of $\mathrm{C}_{18}$ monomers (Kolattukudy, 1996).

Understanding of the types of covalent linkages in plant cutins has been based on the chemical reactivity of the biopolymer. On the other hand, knowledge on the intermolecular cross-linking between cutin monomers has been derived from analysis of the abundance of free primary and free secondary mid-chain hydroxyl functional groups, as well as unesterified carboxyl moieties. Studies involving these approaches demonstrated that about half the mid-chain hydroxyl groups in the biopolymer are involved in ester linkages (Deas and Holloway, 1977). In addition, most of primary hydroxyl groups are involved in ester linkages although there are only a few unesterified carboxyl functional groups (Kolattukudy, 1977).

Structural studies on cutin have been reported by our research group being mainly focused on Fourier-transform infrared (FT-IR) spectroscopical analysis (Ramírez et al., 1992; Luque, 1994) and X-ray diffraction analysis (Luque et al., 1995), which suggested an amorphous structure with basal spacing around $0.45 \mathrm{~nm}$ as repeated unit in the macromolecular structure of cutin. Additionally, solid state ${ }^{13} \mathrm{C}$-nuclear magnetic resonance (NMR) studies of the polyester provided structural information of the intact biopolymer, identi- 
fying distinct polymer domains (Zlotnik-Mazori and Stark, 1988; Garbow and Stark, 1990; Pacchiano et al., 1993). On the other hand, such studies have led the identification and quantification of the principal chemical functionalities of the cutin polymer. Structural and motional characteristics of the major carbon types were also obtained (Garbow and Stark, 1990).

Molecular dynamics (MD) calculations on amorphous polymers have been applied recently. The simulations cover a wide range of different polymers, but molecular modelling of plant biopolyesters has not yet been performed. Computer simulation techniques such as Monte Carlo methods, molecular mechanics (MM) and molecular dynamics (MD) are usually used to model polymeric systems at atomic scales (van Gunsteren and Berendsen, 1990). These methods can provide detailed information that helps to rationalise macroscopically observed behaviour from a microscopic standpoint. On the other hand, MD theoretical simulations can be used to study the qualitative mechanism of penetrant diffusion, its dependence on the parameters of the polymer model used and the strength of the polymer-penetrant interaction (Müller-Plathe, 1992; 1994).

In the present work, the theoretical molecular dynamics of plant cutin has been examined for the first time. The study has been focused on specific points concerning chain molecular flexibility, free molecular volume dependence and other molecular characteristics of the biopolyester. Computational results are also compared with experimental data. In addition, the transport of water across the cutin structure was also studied by MD simulation.

\section{Methods}

\section{Development of the molecular model}

The simulated system consisted of four chains of esterified 10,16-dihydroxyhexadecanoic acid, with a total of 199 atoms. The monomers are linked by ester bonds, which were established between the corresponding carboxyl group and primary and secondary hydroxyl functional groups. A scheme of this initial conformation is showed in Fig. 1A.

The simulation model for cross-linked chain molecules involves several steps through which the different theoretical potentials are calculated.
The energy, $E$, of a system (molecule or polymer) can be described by breaking it into a number of constituent parts (van Gunsteren and Berendsen, 1990):

$$
E=E_{\mathrm{S}}+E_{\mathrm{B}}+E_{\mathrm{T}}+E_{\mathrm{OOP}}+E_{\mathrm{NB}}+E_{\mathrm{ctc}}
$$

Thus the total energy, $E$, is considered to be the addition of a series of individual energies, where $E_{\mathrm{S}}$ is the energy of the bond(s) on being stretched from their "ideal" values; $E_{\mathrm{B}}$, the energy of bending bond angles away from their ideal values; $E_{\mathrm{T}}$, the energy caused by twisting about a bond; $E_{\mathrm{OOP}}$, is the out of plane energy; $E_{\mathrm{NB}}$, the through space or nonbonded energies and $\mathrm{E}_{\mathrm{ctc}}$, other energy terms which individuals may wish to include. Each of these terms may be described by one or more relatively simple expressions. Thus the simplest case for bond stretch energy assumes that Hooke's law describes the stretching of the bond:

$$
E_{\mathrm{S}}=\Sigma_{\text {bonds }} 0.5 k_{\mathrm{S}}\left(d-d_{0}\right)^{2}
$$

where $k_{\mathrm{S}}$ is the energy associated with $d_{0}$, the optimal bond length, and $d$ is the deformed length. Bond angle energy may be described by an expression similar to Hooke's Law:

$$
E_{\mathrm{B}}=\Sigma_{\text {angles }} 0.5 k q\left(\theta-\theta_{0}\right)^{2}
$$

where $\theta$ represent the valence angles in the molecule.

Torsion angle energies require more complex functions, which incorporates cosine values, since $0^{\circ}$ and $360^{\circ}$ must be equivalent, while $180^{\circ}$ and $180^{\circ}$ are equivalent positions, but reached by rotating through different directions. The general method used to describe the changes in energy associated with changes of the torsion angle $\phi$, is by applying the Fourier series:

$$
\begin{aligned}
& E_{\mathrm{T}}=0.5 k_{1}(1+\cos \phi)+0.5 k_{2}(1-\cos 2 \phi)+ \\
& 0.5 \mathrm{k}_{3}(1+\cos 3 \phi)+\ldots
\end{aligned}
$$

where $k$ indicates the different force constants.

Out of plane and other nonbonded energies, for example, van der Waals, salt links and hydrogen bonds are extremely important in determining the conformations of biologically active macromolecules. They may be described by various expressions. The out of plane energies are described by

$$
E_{\mathrm{OOP}}=k_{\mathrm{OOP}} P^{2}
$$


where $P$ is the distance out of plane and $\mathrm{k}_{\mathrm{OOP}}$ the corresponding force constant. This expression is necessary to stop, for example, $s p^{2}$ carbon atoms distorting out of plane, and is often essential in simulations involving peptides and proteins. When two atoms approach one another there is a mutual attraction due to London dispersion forces, but a net van der Waals' repulsion as they get too close, hence at one particular distance, the van der Waals' distance, the attraction is maximal (Leach, 1996). This situation is most simply described by the Lennard-Jones potential (Leach, 1996):

$$
F=\mathrm{A} r^{-12}-\mathrm{Br}^{-6}
$$

where the attraction between atoms $\mathrm{A}$ and $\mathrm{B}$ is described by the $r^{-6}$ and the repulsion by $r^{-12}$. Electrostatic interactions are covered by the familiar equation:

$$
F=\Sigma \mathrm{q}_{1} \mathrm{q}_{2} / \varepsilon \mathrm{r}^{2}
$$

where $q_{1}$ and $q_{2}$ are two ions separated by a distance of $r$ in a medium of dielectric constant $\varepsilon$.

Initial macromolecular conformation was generated by randomly assigning dihedral angles values. Initial bad contacts between different chains and different parts of the same chain were relieved by energy minimisation computation using the Fletcher-Reeves method (Leach, 1996) until a gradient of $0.84 \mathrm{~kJ} / \mathrm{nm}$. The final optimised structure, as shown in Figure 1B, was relaxed enough as not to cause any numerical problems to the subsequent MD calculations.

\section{Molecular dynamics (MD) calculations}

In the MD method a trajectory (or configurations as a function of time) of a molecular system is generated by simultaneous integrations of Newton's equations of motion and for all atoms in the system:

$$
\mathbf{F}=\mathrm{m}\left(d^{2} \mathbf{r} / d t^{2}\right)
$$

where $r$ denotes the position vector of a particle of mass $\mathrm{m}$. MD simulation requires calculation of the gradient of the potential energy $V(\mathbf{r})$. The integration of the above equation is performed in small time steps using an appropriate algorithm (van Gunsteren and Berendsen, 1990).

In this study, MD calculations were carried out subjecting the optimised molecular system repre- sented in Fig. 1B to a calculation of 60 ps duration with a step size of $0.5 \mathrm{fs}$. The temperature was kept constant at $303 \mathrm{~K}$ with a bath relaxation constant of 0.1 ps under vacuum conditions. All simulations were carried out using the HyperChem molecular calculations package (Hypercube, Inc., Waterloo, Canada)). Calculations of a typical 60 ps trajectory required about 38 central processing unit hours.

\section{Diffusion coefficient calculations}

Water molecules were randomly inserted into the macromolecular conformation and equilibrated for $60 \mathrm{ps}$ at $303 \mathrm{~K}$. The system was then equilibrated for $50 \mathrm{ps}$ at the same temperature. From the configurations on the MD trajectory file, the mean-square displacements of the centers of mass of molecules $|R(t)-R(0)|^{2}$ were calculated (Müller-Plathe, 1994). They are averaged over all water molecules and all possible time origins to give the averaged meansquare displacement $<|R(t)-R(0)|^{2}>$.

For long enough times $t$ the least-square displacement becomes linear in time and is given by the Einstein relation (Müller-Plathe, 1994):

$$
<|R(t)-R(0)|^{2}>=6 D t
$$

If the mean-square displacements of the penetrant molecule are plotted against time and leastsquares fit a straight line, the diffusion coefficient $D$ is then calculated (Müller-Plathe, 1994).

\section{Results and Discussion}

\section{Molecular dynamics of the biopolymer cutin}

The common approach modelling a molecular system is a static one. However, a molecular system at any temperature has by no means a static character, and should be described in terms of a multidimensional and complex distribution function of all atomic coordinates and its development in time. The method of MD solves the Newton's equations of motion for a molecular system resulting in trajectories for all atoms in the system. From these trajectories a variety of molecular characteristics can be calculated. Furthermore, three-dimensional structure, and its evolution with time, of the molecular system object of study may be visualised. The start point was the optimised structure showen in Fig. 1B. Fig. 2 shows a series of 

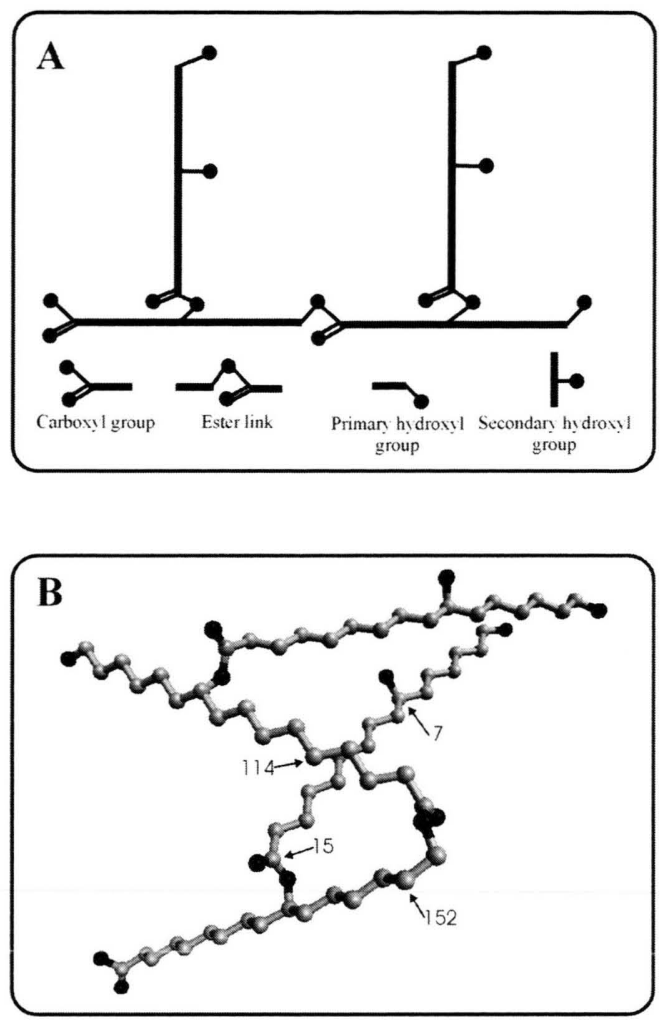

Fig. 1. A. Scheme of the distinct links and functional groups present in the molecular model used for the molecular mechanics (MM) and molecular dynamics (MD) calculations. B. Optimised molecular structure of the cutin model described above. The theoretical polyester was obtained after energy minimisation using the FletcherReeves algorithm (see Leach, 1996) until a gradient of $0.84 \mathrm{~kJ} / \mathrm{nm}$. Hydrogen atoms have been eliminated to clarify the structure. Numbers indicate the exact position of key carbon atoms arbitrarily selected for further molecular dynamics calculations.

snap-shots corresponding to four different structures of the polyester at four distinct times of the simulation. Some differences from the structural point of view can be observed after comparison of the different structures, showing the changing structural character of the modellization.

In order to obtain reasonable structural information, a detailed analysis of the mobilities of carbon-carbon chain and selected atoms was performed. For this purpose, a carbon atom corresponding to a methylene group (carbon number 152, see Fig. 1B) was selected as point of reference of the polymer system. Additional three
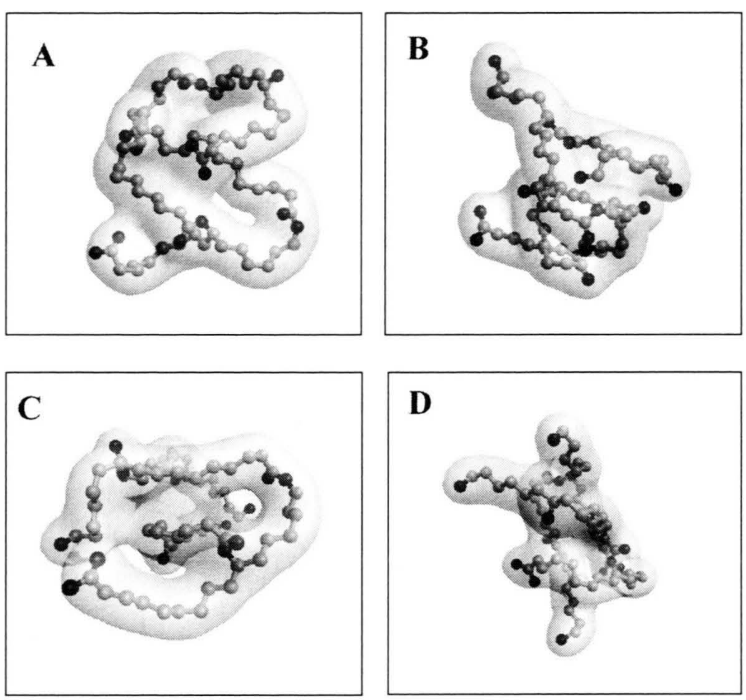

Fig. 2. View of four snapshot pictures of the cutin biopolymer after A) $11 \mathrm{ps}$, B) $25 \mathrm{ps}$, C) $32 \mathrm{ps}$ and D) $41 \mathrm{ps}$ of time simulation equilibrated at $303 \mathrm{~K}$. Grey volume represents the van der Waals surface of the different chains that form the biopolymer. Oxygen atoms are showed in black, carbon atoms in light grey. Hydrogen atoms have been eliminated to improve the clarity of the image. Note how the chain segments folding $(B, D)$ and spread (A, C) allowing the formation of intramolecular cavities or holes.

atoms of carbon were selected belonging to a secondary alcohol group $(\mathbf{C H O H}$, atom number 7, Fig. 1B), ester group (CHOCOR, atom number 15, Fig. 1b) and to a methylene group $\left(\mathbf{C H}_{2}\right.$, atom number 114, Fig. 1b). The root mean square (RMS) mean-deviation function $\mathrm{D} x=$ $\left(<x^{2}>-<x^{2}>\right)^{1 / 2}$ where $<x^{2}>=\left(1 / n \sum x_{\mathrm{i}}^{2}\right)$ and $<x^{2}>=$ $\left(1 / n \sum x_{\mathrm{i}}\right)^{2}$ of the relative distance $x$ from the reference atom was used to obtain quantitative data. Fig. 3 shows a plot of the distances for the three selected carbon atoms above defined as a function of time. It is interesting to point out that the analysis resolves three different motional behaviours of the selected carbon atoms. Methylene carbon, $\mathrm{CH}_{2}$, appeared the less mobile, followed by the carbon located at the ester bond (CHOCOR), which is the carbon atom that supports the secondary hydroxyl functional group, $(\mathbf{C H O H})$, the most mobile one. The mobility ratio between the three different groups was 1:2.5:5, respectively. These results agree well with previously experimental data 


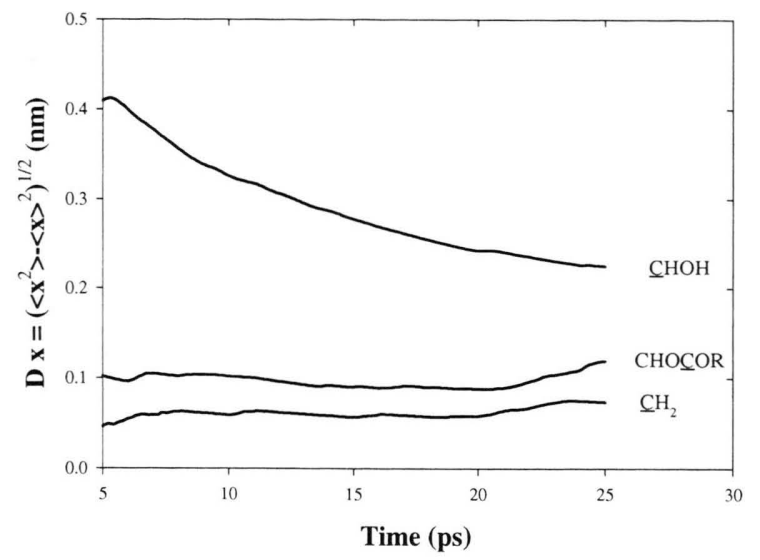

Fig. 3. Displacement of selected carbon atoms belonging to methylene chain, secondary hydroxyl group and ester group of the cutin molecular model showed in Figure 1 from their initial positions versus time. MD calculations have been made at the temperature constant of $303 \mathrm{~K}$. The graphs represent the root mean square (RMS) of the relative distance for three different selected carbon atoms of the cutin structure from the reference atom (see Fig. 1.B) as a function of computation time.

based on ${ }^{13} \mathrm{C}$ - nuclear magnetic resonance study of isolated fruit lima cutin (Zlotnik-Mazori and Stark, 1988). These authors discussed a dynamics profile of the structural components of the plant cuticle in terms of a flexible network mainly constrained by specific cross-links located at the secondary alcohol esters.

An interesting point on the modellization analysis is related to the three-dimensional dynamic structures depicted in Fig. 2. The motion of the different chains and atoms conduce the biopolymer to a self-folding or assembly keeping the ester links and the most polar functional groups outside the macromolecule. This leads to two important conclusions. First, the existence of molecular spacings between the polymer chains. Second, and consequently, the continuous formation of holes and cavities within the structure. It is important to emphazise that the average distance between the polymeric chains, measured in the different structures obtained, was around $0.4-0.5 \mathrm{~nm}$ which agrees well to the basal distance measured by Xray diffraction on tomato fruit cutin and others plant cutins (Luque, 1994; Luque et al., 1995). Thus, the molecular modelling draws a picture of plant cutin according to their amorphous and hydrophobic characteristics.

\section{Molecular diffusion of water molecules. MD calculations}

The practical application of selective gas permeation through polymer membranes has generated great interest in theoretical models and methods to study underlying molecular mechanisms. Quantitative predictions of permeability and selectivity open the prospect to develop tools for the design of membranes with predefined properties (MüllerPlathe, 1994; Leach, 1996). As pointed out in the introduction, MD simulations are an interesting and valuable tool to study diffusion processes. They have been intensively used to study diffusion in synthetic polymers as polyethylene and polypropylene (Müller-Plathe, 1991, 1992). Water-cuticle interaction and diffusion is an old topic in cuticle research because of its physiological importance and significance (for a recent review see Kerstiens, 1996). In spite of its importance, the exact mechanism of the water diffusion through plant cuticular membranes still remains to be clarified (Schönherr, 1982; Luque et al., 1995; Niederl et al., 1998).

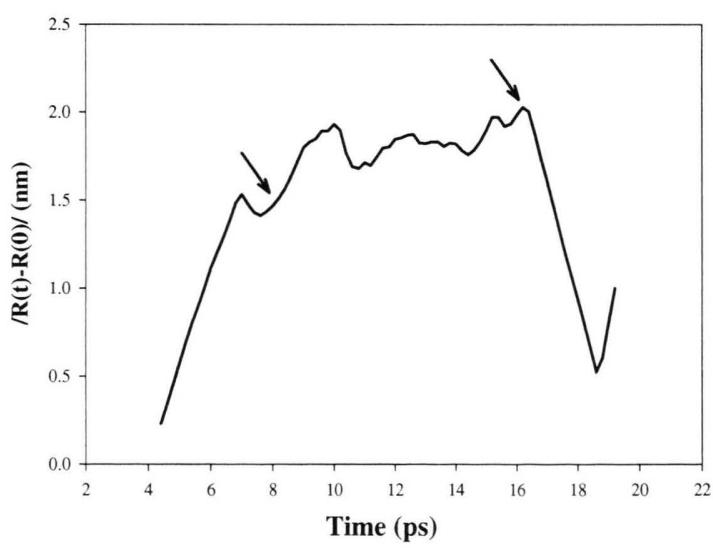

Fig. 4. Theoretical displacement of a water molecule into the host cutin structure as function of time of computation. The theoretical displacement is represented by the average displacement of the center of mass of the water molecule from the time origen (see Methods). Arrows indicate the time interval for water molecule oscillations around its equilibrium position. From data between this time interval and applying Eq. 9, the theoretical diffusion coefficient can be calculated. For more details see text. 
Previous applications of MD simulations to the diffusion process have established that the underlying process is a "hopping mechanism" (Müller-Plathe, 1994). For a considerable simulation time, the penetrant molecule, water in our case, is located in molecular holes or voids present in the structure of amorphous polymers. In that state they essentially perform oscillations only around their equilibrium positions.

As indicated in Methods, a useful quantity for qualitative studies of the permeation and motion pattern of a penetrant gas molecule during a simulation is the displacement from its initial position, $|R(t)-R(0)|$. Fig. 4 shows the displacement of a selected water molecule in the cutin polymer from 2 until 20 ps of simulation time. This water molecule has been selected as a good example of the MD diffusion simulation. Inspection of the figure clearly confirms that for this water molecule the hopping mechanism prevails. This process occurred between 8 and 16 ps of the simulation (see arrows in Fig. 4) where the water molecule performed small oscillations, about only $0.2 \mathrm{~nm}$, around its equilibrium position. In this case, the host polymer (the cutin) retained the penetrant small molecule (water). Above this time, the molecular displacement suffered again noticeable variations (Fig. 4). This molecular event is better observed in the Fig. 5 where a three-dimensional view of the trajectory of the water molecule into the cutin polymer is shown. Arrows indicate the exact time when the water molecule jumps into the hole.

According to the Einstein equation (Eqn. 9), the diffusion coefficient calculated from the mean-squared displacements for this one-particle trajectory was of $1.89 \times 10^{-9} \mathrm{~m}^{2} \mathrm{~s}^{-1}$ at $303 \mathrm{~K}$. This value can be compared to the experimental values reported by Kerstiens (1996) for the cuticular water permeance or minimum conductances to water vapour of leaves. These values range from 0.1 to $100 \times 10^{-5} \mathrm{~m} \mathrm{~s}^{-1}$ (Kerstiens, 1996). If we assume a layer thickness of $1 \mu \mathrm{m}$, and consider a homogenous layer, we could obtain apparent experimental diffusion coefficients of

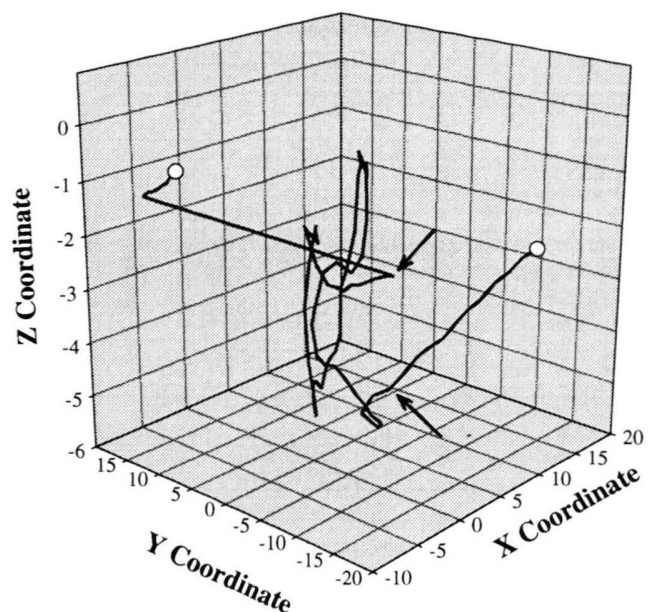

Fig. 5. Trajectory of a water molecule during the first 20 ps of the MD simulation at $303 \mathrm{~K}$. Note the tortuousity of the trajectory and how the diffusion pathway is far to be considered linear. Arrows indicate the time and position when the water molecule interacts with the cutin network, just before the water molecule (represented by open circles) jumps into the hole (for more details see text).

the same order of magnitude as the theoretical value derived by this work.

In this study we made a first theoretical approach to the structure and molecular arrangement in a short chain biopolymer, the plant cutin, using molecular dynamics computer simulations. Although the model has its limitation, the data of the theoretical model obtained within the time scale of usual MD simulations agrees well with structural and spectroscopic experimental data of the plant cutin. The diffusion of water vapour as penetrant molecule through the cutin has been also studied. Qualitatively, the diffusion proceeds by the hopping mechanism mentioned, i.e., the water molecules oscillate for longer periods of time around certain positions and perform quick jumps between these positions. The data described open a new and exciting field of research which can assist studies devoted to molecular aspects of the plant cuticle to improve our understanding of this important plant biopolymer. 
Deas A. H. B. and Holloway P. J. (1977), The intermolecular structure of some plant cutins. In: Lipids and lipids polymer in higher plants (Tevini M. and Lichtenthaler H. K., eds.). Springer-Verlag, Berlin, 293300.

Garbow J.R and Stark R. E. (1990), Nuclear magnetic resonance relaxation studies of plant polyester dynamics. 1. Cutin from limes. Macromolecules 23, $2814-2819$.

Holloway P. J. (1982), Structure and histochemistry of plant cuticular membrane: an overview. In: The Plant Cuticle ( Cutler D. F., Alvin K. L., Price C. E., eds.). Academic Press, London, UK, 1-32.

Leach A. R. (1996), Molecular Modelling. Principles and Applications. Addison Wesley Longman Ltd., London.

Luque P. (1994), Propiedades fisicoquímicas y estructura de la cutícula de fruto de tomate (Lycopersicon esculentum L.) Ph. D. Thesis. Universidad de Málaga.

Luque P., Bruque S. and Heredia A. (1995), Water permeability of isolated cuticular membranes: a structural analysis. Arch. Biochem. Biophys. 317, 417-422.

Kerstiens G. (1996), Cuticular water permeability and its physiological significance. J. Exp. Bot. 47, 1813-1832.

Kolattukudy P. E. (1996), Biosynthetic pathways of cutin and waxes, and their sensitivity to environmental stresses. In: Plant cuticles. An integrated functional approach Kerstiens G., de.). Bios Sci. Publ., Oxford, $83-108$.

Müller-Plathe F. (1991), Diffusion of penetrants in amorphous polymers: A molecular dynamics study. J. Chem. Phys. 94, 3192-3199.
Müller-Plathe F. (1992), Molecular dynamics simulation of gas transport in amorphous polypropylene. J. Chem. Phys. 96, 3200-3205.

Müller-Plathe F. (1994), Permeation of polymers. A computational approach. Acta Polymer 45, 259-293.

Niederl S., Kirsch T., Riederer M. and Schreiber L. (1998), Co-permeability of ${ }^{3} \mathrm{H}$ - labeled water and ${ }^{14} \mathrm{C}$ labeled organic acids across isolated plant cuticles. Plant Physiol. 116, 117-123.

Pacchiano R. A., Sohn W., Chlanda V. L., Garbow J. R. and Stark R. E. (1993), Isolation and spectral characterisation of plant-cuticle polyesters. J. Agric. Food Chem. 41, 78-83.

Ramírez F. J., Luque P., Bukovac M. J., and Heredia A. (1992), Fourier transform IR study of enzymatically isolated tomato fruit cuticular membrane. Biopolymers 32, $1425-1429$.

Schönherr J. (1982), Resistance of plant surfaces to water loss: transport properties of cutin, suberin and associated lipids. In: Encyclopedia of Plant Physiology, Vol. 12B, Physiological Plant Ecology 2 (Lange O. L., Nobel P. S., Osmond C. B., Ziegler H., de.). Springer-Verlag, Berlin, 153-179.

van Gunsteren W. F. and Berendsen J. C. (1990), Computer simulation of molecular dynamics: methodology, applications and perspectives in chemistry. Angew. Chem. Int. Ed. Engl. 29, $992-1023$.

Walton T. J. (1990), Waxes, cutin and suberin. Meth. Plant Biochem. 4, 105-158.

Zlotnik-Mazori T. and Stark R. E. (1988), Nuclear magnetic resonance studies of cutin, an insoluble plant polyester. Macromolecules 21, 2412-2417. 